\title{
Challenges of pain control and the role of the ambulatory pain specialist in the outpatient surgery setting
}

\author{
This article was published in the following Dove Press journal: \\ Journal of Pain Research \\ 17 June 2016 \\ Number of times this article has been viewed
}

\section{Nalini Vadivelu' \\ Alice $\mathrm{M} \mathrm{Kai}{ }^{2}$ \\ Vijay Kodumudi ${ }^{3}$ \\ Jack M Berger ${ }^{4}$}

'Department of Anesthesiology, Yale University School of Medicine, New Haven, CT, ${ }^{2}$ Stony Brook University School of Medicine, Stony Brook, NY, ${ }^{3}$ Department of Molecular and Cell Biology, College of Liberal Arts and Sciences, University of Connecticut, Storrs, CT, ${ }^{4}$ Department of Anesthesiology, Keck School of Medicine, University of Southern California, Los Angeles, CA, USA

Correspondence: Nalini Vadivelu Department of Anesthesiology, Yale University School of Medicine, TMP 3, 333 Cedar Street, New Haven, CT 06519 , USA

Tel +l 2037852802

$\mathrm{Fax}+\mathrm{I} 2037856664$

Email Nalini.vadivelu@yale.edu
Abstract: Ambulatory surgery is on the rise, with an unmet need for optimum pain control in ambulatory surgery centers worldwide. It is important that there is a proportionate increase in the availability of acute pain-management services to match the rapid rise of clinical patient load with pain issues in the ambulatory surgery setting. Focus on ambulatory pain control with its special challenges is vital to achieve optimum pain control and prevent morbidity and mortality. Management of perioperative pain in the ambulatory surgery setting is becoming increasingly complex, and requires the employment of a multimodal approach and interventions facilitated by ambulatory surgery pain specialists, which is a new concept. A focused ambulatory pain specialist on site at each ambulatory surgery center, in addition to providing safe anesthesia, could intervene early once problematic pain issues are recognized, thus preventing emergency room visits, as well as readmissions for uncontrolled pain. This paper reviews methods of acute-pain management in the ambulatory setting with risk stratification, the utilization of multimodal interventions, including pharmacological and nonpharmacological options, opioids, nonopioids, and various routes with the goal of preventing delayed discharge and unexpected hospital admissions after ambulatory surgery. Continued research and investigation in the area of pain management with outcome studies in acute surgically inflicted pain in patients with underlying chronic pain treated with opioids and the pattern and predictive factors for pain in the ambulatory surgical setting is needed.

Keywords: outpatient surgery analgesia, ambulatory surgery pain control, acute pain, ambulatory surgery center, opioids, substance abuse

\section{Introduction}

Pain is a critical component of patient management that often not only consists of physical disability but also necessitates care to address the psychological, emotional, and economic strain it places on patients and their caretakers. It places a significant economic strain on society in the form of disability, loss in productivity, and health care costs. ${ }^{1}$ Moderate-to-severe pain continues to be experienced by up to 70 percent of patients after surgery. ${ }^{2-4}$ Optimum management of pain is crucial in the outpatient as well as in the inpatient surgery setting. Ambulatory surgery is on the rise in the US. In fact, there has been more than $100 \%$ increase in the number of ambulatory surgery centers since 1990 in the US. ${ }^{5}$ Perioperative pain control is vital, since ineffective pain control can lead to increased morbidity and mortality. ${ }^{6}$ Optimum pain control in the ambulatory setting is complex and challenging, and includes the implementation of interventions to manage and prevent flare-ups in patients with a history of preexisting chronic pain and in those with a history of substance abuse. Uncontrolled pain 
and nausea are the two most common reasons for delayed discharge and unexpected admissions to the hospital, necessitating the focus on control of pain in the ambulatory setting leading to an implementation of opioid-sparing analgesia in ambulatory surgery. ${ }^{7-9}$

Nausea and respiratory depression are common side effects of pain medications, so prevention and management of side effects of nausea and respiratory depression are essential as well. The challenges of pain control in ambulatory pain control have become increasingly recognized in recent times, ${ }^{1,2,10}$ and research on solutions to provide adequate pain control in ambulatory surgery centers is ongoing.

\section{Challenges for the ambulatory care pain specialist}

It is important to provide safe and optimum pain management for ambulatory surgery patients preoperatively, intraoperatively, and postoperatively for their acute-pain issues, ensure prevention of pain flare-ups and withdrawal syndromes, and ensure safe discharge from the ambulatory surgery center. Effective analgesia can often be achieved with medications causing less nausea and/or with utilization of regional anesthesia techniques in conjunction with nonopioid therapies. Traditional sections of the division of a pain service in medical centers include acute-pain service, regional or block service, and a chronic-pain service, but not an ambulatory pain service in ambulatory surgery centers. These services could be facilitated by the presence of an ambulatory care pain specialist overseeing a clinical program for pain control in every ambulatory surgery center, who is a practicing anesthesiologist on site with a special interest in pain management at the ambulatory surgery center, which is a new concept. By virtue of clinical training, orientation, and experience through daily clinical practice at the ambulatory surgery center, the ambulatory pain-management specialist would be particularly suited to address the unmet needs of ambulatory pain management in the unique setting of ambulatory surgery, where the majority of surgery is performed in the US. The ambulatory pain-management specialist could typically take care of patients in the ambulatory surgery center, providing safe anesthesia to patients with a history of pain-control issues, substance abuse, and chronic pain. The ambulatory pain specialist would work to provide safe and optimum pain management preoperatively, intraoperatively, and postoperatively for acute-pain issues of patients at the same time, preventing pain flare-ups and withdrawal syndromes, thereby ensuring safe discharge from the ambulatory surgery center.

In addition to having direct oversight of clinical trainees in the operating rooms and being a resource offering consultation for optimum perioperative pain control for patients undergoing ambulatory surgery, the ambulatory pain specialist would be in charge of the clinical program of ambulatory pain control in each ambulatory center, oversee quality-ofcare initiatives to intervene early, oversee implementation of a treatment plan for pain at the ambulatory surgery center in patients with complex pain issues, and prompt intervention to control nausea and vomiting and prevention of pain flareups and withdrawal symptoms in patients who are opioiddependent and taking part in substance-abuse programs. The ambulatory pain specialist would also oversee the formulation of clinical protocols in an ambulatory postanesthesia care unit (PACU), which is designed to improve teaching as well as patient satisfaction.

Setting standards of care for the treatment of pain at the ambulatory surgery center by the pain specialist would include the identification of patients at increased risk of perioperative pain and the implementation of a broad and cost-effective multimodal analgesia protocol, as well as instituting other rescue interventions when the implemented plan falls short. Goals are to be identified. These could include preoperative chronic pain, reduction of opioid exposure, prevention of hyperalgesia, and the minimization of side effects and risks from nonopioids. Laying out plans for additional analgesics in the recovery room, rescue blockage, and postoperative regional anesthetic blocks would be helpful. Additional preoperative and postoperative contingencies may be employed for patients with higher than baseline risk. Gerbershagen et al studied whether the severity of preoperative chronic pain was a good predictor of acute postoperative pain in patients who had undergone radical prostatectomy. ${ }^{11}$ The study observed that patients with greater preoperative chronic pain complained of more intense acute pain postoperatively. Additional contingencies could include intravenous acetaminophen, increased-potency opioids, such as oxycodone, or supplemental regional techniques for patients with uncontrolled pain or opioid tolerance, where a two- to threefold increase in opioid requirement can be seen.

Pain-management medications are often abused, which complicates the management of pain in the ambulatory setting. Substance abuse and opioid addiction is on the rise, and patients with substance abuse can pose a supreme challenge 
for pain control. Prescription pain-management medications are often abused and used nonmedically, which complicates the management of pain in the ambulatory setting. The abuse of prescription drugs, such as opioids, stimulants, such as amphetamine-dextroamphetamine and methylphenidate, and central nervous system depressants, such as benzodiazepines, has grown to epidemic proportions. ${ }^{12,13}$

An ambulatory care pain specialist could ensure that patients with a history of substance abuse and opioid dependence who are undergoing treatment for substance abuse (such as methadone and buprenorphine) do not develop substance-withdrawal syndromes or pain flare-ups, while receiving optimum pain control, thereby facilitating safe discharge. Long-term administration of opioids may necessitate an increase in the dosage of opioids needed to maintain the appropriate level of analgesia.

Studies with human subjects suggest that patients who are maintained on methadone are hyperalgesic in comparison to individuals who were previously addicted ${ }^{14}$ and significantly more hyperalgesic than control subjects. ${ }^{15,16} \mathrm{An}$ increasing number of studies suggest that long-term use of opioids causes changes in pain sensitivity. It has been seen that patients abusing or addicted to opioids presenting for ambulatory surgery will often have coexisting psychiatric disorders. For instance, patients addicted to heroin have an increased prevalence of personality disorders, anxiety, psychosis, and depression. ${ }^{17,18}$

The ambulatory pain specialist would be available for consultation by surgeons and anesthesiologists on a daily

Table I Role of the ambulatory pain specialist overseeing the clinical program of ambulatory pain control

I. Develop standards of care for treatment of pain at the ambulatory surgery center

2. Identification of patients at risk for increased pain

3. Provide consultation for advanced pain care at the ambulatory surgery center

4. Pain control in patients with history of substance abuse to prevent flare-up of pain and withdrawal syndromes

5. Utilization of early rescue techniques for pain control

6. Implementation of multimodal analgesia

Multiple medications acting at different sites on the pain pathway and different mechanisms of action

Medications include nonopioids, opioids, $\alpha_{2} \delta$-modulators, and corticosteroids

Multiple techniques, such as local anesthesia infiltration, regional anesthesia techniques, peripheral nerve-block catheters

7. Control of nausea and vomiting

8. Planning of discharge medications, weaning, and follow-up for control of pain by primary care providers basis to review cases and assist in providing safe and optimum pain management for all patients. The ambulatory care specialist would also be responsible for advanced pain care in both the preoperative holding areas and PACUs. Endoscopic procedures, such as gastroduodenoscopy, ultrasound, colonoscopies, and endoscopic retrograde cholangiopancreatography performed for stones or for cancer of the gastrointestinal system, such as pancreatic cancer, and other imaging studies are now often performed as outpatient procedures in patients with painful states, potentially increasing the need for ambulatory pain specialists and pain control in ambulatory surgery centers. Advanced pain care and multidisciplinary communication and coordination may be needed in patients with implanted intrathecal drug-delivery systems, which deliver intrathecal opioids and other medications, as well as patients with implanted dorsal column stimulators who are to undergo surgery in the ambulatory surgery center with a history of pain-control issues, substance abuse, and chronic pain. This systematic approach in the ambulatory surgery center would decrease the rate of delayed discharge from the PACU and unanticipated hospital admissions, and improve pain outcomes in ambulatory surgery centers (Table 1).

\section{Real-world challenges in implementing the change to include the role of the ambulatory pain specialist}

The implementation of the change to integrate an ambulatory pain specialist is associated with real-world challenges. The integration of an ambulatory pain specialist into an ambulatory surgery center is a necessary but not an easy task. It involves clinical, economic, and noneconomic integration. This would entail detailed planning, assessment of human resources, and financial considerations by physicians and hospital management. The clinical integration of any new clinical program, such as the integration of an ambulatory pain specialist, would need good hospital-physician relationships, be executed with care as a collaborative arrangement, and with evaluation of every minute detail, including discussions on cost of staffing, acquisition of special training requirements, and skills. ${ }^{19}$

The integration of an ambulatory pain could also potentially impact on the requirement of future skills needed in ambulatory or general anesthesiologists to provide optimum postoperative pain control, with the current shift toward greater numbers of patients undergoing surgery as outpatients than inpatients. 
In addition, the feasibility of a single pain specialist to provide the bandwidth for all advanced regional anesthesia and pain planning in an ambulatory surgery center needs to be analyzed for each ambulatory center. Perhaps the ambulatory pain specialist in larger ambulatory surgery centers would need assistance from other anesthesiologists in the group with a special interest in pain control as well.

\section{Pain is subjective}

It is important to work to develop a systematic approach to the subjective phenomenon of pain in the ambulatory surgery center. The International Association for the Study of Pain states that actual or potential damage to tissues can lead to emotional and sensory experiences. ${ }^{20}$ It is important then to understand that both sensory and emotional experiences that are unpleasant need to be addressed. Pain is subjective, with varying thresholds in different individuals. Pain appears to have a sociopsychobiological perspective, and it is important to understand this to achieve optimum pain control. ${ }^{21}$

Unpleasant sensory and emotional experiences (pain) are felt with the same afferent nociceptive impulse, which in the somatosensory cortex enables the patient to feel the sensation of pain while eliciting an emotional response at the limbic structures. ${ }^{22}$ There could thus be a demarcation between nociception, pain, and suffering according to the biopsychological model of pain proposed by Mack et al. ${ }^{23}$

It is important also to make the distinction between several pain states that are encountered in patients presenting for surgery in the ambulatory care setting. Pain control depends greatly on individual pain states, the pain characteristics, and the injury or surgical insult, all of which result in making the experience of pain a very subjective phenomenon. ${ }^{24}$

\section{Postoperative pain is a common reason for unanticipated hospital admission after outpatient surgery}

It is important to understand the pattern and predicting factors of postoperative pain in the ambulatory surgery setting, ${ }^{25}$ in order to provide optimal pain control and prevent delayed discharges and unanticipated hospital admission. The pattern and predicting factors for severe pain were studied in a prospective study involving 10,008 consecutive ambulatory surgical patients. It was seen that certain types of surgery and body mass index were significant predictors of pain in the recovery room. For example, of the surgeries studied, orthopedic patients had the highest incidence of severe pain, at $16.1 \%$, while urologic, general, and plastic surgery had $13.4 \%, 11.5 \%$, and $10 \%$, respectively. Younger males, those with a higher body mass index, and patients with longer duration of anesthesia also had a higher incidence of severe pain. The duration of stay in the recovery room and longer time to discharge from the recovery room were also seen in patients with severe pain. ${ }^{25}$ Preoperative screening for risk stratification should include the pattern and predictive factors of postoperative pain in ambulatory surgery while developing a pain-treatment plan for prophylactic analgesic regimens and other analgesic approaches. In order to prevent delayed discharges and unanticipated hospital admissions, an opioid-sparing analgesic approach has also been suggested..$^{7-9}$

\section{Assessment and treatment planning according to risk for pain control in ambulatory surgery}

A detailed preoperative pain history is extremely important for risk stratification and planning-treatment strategies to deliver optimal pain control perioperatively in the ambulatory setting. ${ }^{24,26}$ It is ideal to obtain this information a few days earlier than the planned surgery, and a thoughtful pain questionnaire would make it easy to gather this information for the patient and the caregivers. This questionnaire could include questions on site of pain, its frequency, durations of pain, types of medications, and dosages taken for the pain. Additional questions on history of substance abuse, depression and anxiety, sleep issues, poor pain control, and patient expectations, and patient caregivers would help to risk-stratify patients and develop a plan for treatment of pain preoperatively, intraoperatively, and postoperatively in the ambulatory surgery center. This questionnaire should be directed to enable the health care practitioner to get a sense of aberrant pain states due to abnormal processing, such as tolerance, substance abuse, partial-agonist therapy, history of chronic pain, and uncontrolled postoperative pain in the past, which would help in customized treatment planning to provide optimal pain relief perioperatively for each patient. It is advisable that the ambulatory pain specialist confer with the surgical team, the patients, and their families, as well as medical personnel, to gather input from all concerned to make a cohesive and effective plan for effective pain control before implementation of the pain-treatment plan in the ambulatory surgery center.

Once the risk of postoperative uncontrolled pain is determined, the next step would be to develop a plan for the provision of effective multimodal analgesia. To achieve optimum pain management, it is important first to determine the expectation of the patients in the form of goals to enable the caregiver to work closely with their patients 
to obtain patient satisfaction. These goals often could be to decrease risks associated with nonopioids, as well as to reduce the exposure to opioids, prevention of hyperalgesia, availability of additional analgesics for breakthrough pain, and implementation of regional anesthesia preoperatively or postoperatively.

\section{Implementation of effective multimodal analgesic interventions and interventions for pain rescue}

As per American Society of Anesthesiologists recommendations, patients having ambulatory surgery could take oral nonopioids, such as acetaminophen, gabapentin, and diclofenac or celecoxib with a sip of water before surgery to decrease the need for intravenous medications. These oral premedications combined with a regional anesthetic technique where applicable can result in optimum pain control. In addition to providing a safe anesthetic in the ambulatory surgery center, the health care providers should incorporate multimodal analgesia to provide a short and comfortable recovery-room stay, facilitate early discharge rehabilitation, and improve home recovery. The pain treatment, in addition to the implementation of multimodal analgesic interventions that would include rescue interventions, in the ambulatory care setting is often necessary to achieve optimum pain relief depending on the complexities of the pain problems, patient comorbidities, the type of surgery, and patient expectations. It is also always important to consider the cost of the interventions that may be needed after implementation of multimodal analgesic and rescue interventions to make the strategies viable in the long term in the ambulatory surgery center. Costs include services by personnel and supplies. ${ }^{26,27}$ It is important, however, to consider lowering costs without sacrificing patient safety. Appropriate control of acute pain with multimodal techniques would preemptively mitigate the development of chronic pain. There are medical and economic burdens associated with the transition of acute to chronic pain and the severity of chronic pain. Chronic pain leads to decreased functionality, greater work absences, and associated conditions, such as depression. ${ }^{28}$ In addition, chronic pain also impacts quality of life, with escalation of costs and utilization of health care resources. ${ }^{29}$ It is thus compelling to prevent chronic pain preemptively with optimum control of acute pain with multimodal approaches.

\section{Interventions to control anxiety}

Interventions to control severe anxiety can be with medications such as gabapentinoids preoperatively and benzodiazepines perioperatively. ${ }^{30}$ It is also worth noting that psychological factors also play a large role in the development of chronic pain following surgery. ${ }^{31,32}$ The National Center for Health Statistics in $2006^{33}$ approximated that about a quarter of the population in the US experience recurring or chronic pain, of which $40 \%$ report suffering moderate-to-severe degrading impact. The majority of patients with chronic pain complain of multiple foci of pain. Rates of chronic pain increase with advancing age, ranging from $25 \%$ to $50 \% \cdot{ }^{34} \mathrm{~A}$ number of factors predict worse pain control and amplified analgesic demands in postoperative patients: psychological states, such as neuroticism, depression, and anxiety; age and sex; preoperative opioid use; and preexisting conditions of pain. ${ }^{35,36}$ Furthermore, some of the psychological conditions, namely depression, have been found to increase the probability of heightened perioperative pain experienced and opioid consumption. ${ }^{37}$

\section{Interventions in patients with history of chronic pain}

It has been approximated that in the US, chronic pain not only affects functionality but is also a significant socioeconomic health problem, with an annual cost estimated to be as high as $\$ 100$ billion. ${ }^{1}$ There remains a dispute and lack of general agreement about the utilization of opioids for treating chronic nonmalignant pain.

The majority of the debate revolves around reservations regarding the long-term analgesic efficacy of opioids, the risk of side effects, potential for abuse, and dependence, and the capability of physicians in determining suitable patients to receive this form of treatment. ${ }^{3}$ Regardless, numerous guidelines for the treatment of chronic nonmalignant pain include opioids as a choice for therapy, particularly if more conventional treatment options have been ineffective. ${ }^{38-40}$

At present, approximately two-thirds of all surgeries are performed in the ambulatory care setting. With the increase in the volume of ambulatory surgeries, there has been an increase in the number of patients with a history of chronic pain presenting for surgery in ambulatory surgery centers as well. Though most studies on the management of perioperative pain in patients with a history of chronic pain have been done in the inpatient setting, similar challenges could be faced in the management of chronic-pain patients in the ambulatory surgery setting.

The management of acute surgically inflicted pain in patients with underlying chronic pain treated with opioids is an area of pain management that needs to addressed and investigated. Gerbershagen et $\mathrm{al}^{11}$ studied whether the 
severity of preoperative chronic pain was a good predictor of acute postoperative pain in patients who had undergone radical prostatectomy. The study observed that patients with greater preoperative chronic pain complained of more intense acute pain postoperatively. With the belief and consideration that opioid drugs can help alleviate chronic pain, recover mood, and help recover daily functionality, many physicians have encouraged opioid pharmacotherapy for the management of chronic pain. In part because alternative methods for chronic-pain management remain limited, the utilization of opioid pharmacotherapy has outpaced the advance and progress of studies substantiating the benefits of opioid pharmacotherapy. ${ }^{41}$ A number of studies have been conducted that suggest that the use of opioids can cause perioperative hyperalgesia and overall increased animal and human sensitivity to noxious insult, ${ }^{38,42,43}$ while an evidence-based literature review regarding opioid-induced hyperalgesia was inconclusive. ${ }^{44}$

Chapman et $\mathrm{a}^{45}$ studied the severity of postoperative pain for 6 days in two groups of patients: those with preexisting chronic pain with opioid pharmacotherapy, and a matched control group, both undergoing general surgery and orthopedic surgery. The study found that the group of patients with preexisting chronic pain using opioids reported considerably greater levels of postoperative pain than the second group, and they also experienced a relatively delayed rate of resolution of the pain. A follow-up observational study followed 55 patients with preexisting chronic pain 2 weeks following surgery, of which 25 subjects did not utilize opioid pharmacotherapy and 30 subjects did. ${ }^{42}$ Daily postoperative pain with rest and movement was reported. The study found that there was a substantially greater level of pain throughout the study among the patients on opioid pharmacotherapy. There was no significant difference between the rates of pain resolution between the two groups of chronic-pain patients. The findings of this study thus supported evidence provided by animal studies that opioid-induced hyperalgesia is produced by the extended use of opioids. ${ }^{46,47}$ Patients suffering from chronic pain therefore require distinctive consideration for the management of their postoperative pain when they undergo surgical procedures.

Greater postoperative pain has been associated with a greater risk of chronic pain as a result of surgery. ${ }^{48}$ Consequently, aggressive management of postoperative pain may be particularly critical in patients with chronic pain, since they characterize a group that is especially at risk for and susceptible to complications and chronic postoperative pain. ${ }^{38}$

Patients with chronic pain are frequently treated preoperatively with COX inhibitors, opioids, coanalgesics, anticonvulsants, and antidepressants. Many of them have extended sedentary lifestyles and neurological deficits that may be associated with the adverse events, and complications of perioperative management, due to a combination of drug interactions, side effects, and issues with tolerability and tolerance. Overadministration and unsuitable doses of drugs are frequently seen issues among chronic-pain patients. ${ }^{49} \mathrm{Often}$, these patients also have decreased functionality of various organ systems, and are thus at greater risk for adverse physiological reactions of the neuroendocrine system to pain. ${ }^{50} \mathrm{In}$ addition, patients suffering from chronic pain often underreport and undervalue their usage of drugs. This propensity is more predominantly observed in female patients and in patients taking opioid analgesics in comparison to other drugs. ${ }^{51}$ Caretakers may also tend to overemphasize addiction, tolerance, and sedation due to analgesics, but underestimate the effect of COX inhibitors and coanalgesics on pain. ${ }^{52}$ On the day of the procedure, some patients may neglect to take their daily opioid dose, either due to instructions to not take medications on the day of the surgery or because they erroneously think that they should not take their oral opioid dose since oral intake of liquids and solids are discouraged.

Giving partial-opioid agonists, including nalbuphine or buprenorphine, to patients chronically on opioids may cause sudden opioid withdrawals and should be avoided. Though minimal doses of partial-opioid agonists are helpful in patients who are opioid-naïve for the purpose of relieving side effects, such as nausea and pruritus, commonly caused by full agonists, their utilization in chronic-pain patients dependent on opioids remains complex and necessitates close observation and constant management. ${ }^{38}$ It would be prudent to consider inpatient admission for complicated opioid-dependent patients to determine the amount of oral opioid necessary to control their pain.

\section{Interventions in chronically opioid- consuming patients}

There is a dearth of information on the management of patients chronically on opioids perioperatively when presenting for surgery in the ambulatory setting. Preoperatively, the preoperative clinic personnel, surgical team, and anesthesia team participating in the care of the patients should identify patients who are administered opioids chronically. Since the lowest daily opioid dose that appreciably increases postoperative requirements for opioids remains undetermined, all patients should be educated about the possibility of increased opioid needs and intensified pain postoperatively. Furthermore, they should be notified of other available analgesic 
methods that complement opioids. Patients who chronically consume opioids should not forgo their daily dose of opioid on the day of the procedure, particularly if significant doses of long-acting opioids are regularly taken, in order to prevent withdrawal. $^{38}$

However, it goes without question that the patient will need their preoperative opioid dose or opioid equivalent as the starting dosage to which additional opioid must be added according to the patient's need to avoid withdrawal and to cover the nociception created by the surgical intervention. ${ }^{38}$ This could be as much as three times higher than the expected opioid dose in nonchronic pain (opioid-naïve) patients. ${ }^{35}$ It must be remembered that epidural opioids do not cover the systemic need created by chronic oral opioid administration.

Underreporting the usage of drugs is more predominate in female patients and in patients taking opioid analgesics in comparison to other drugs. ${ }^{51}$ As a result, inadequate management perioperatively may go overlooked and may cause withdrawal. ${ }^{50}$ Preoperative history-taking by the caretakers may also tend to overemphasize addiction, tolerance, and sedation due to analgesics, but underestimate the effect of COX inhibitors and coanalgesics on pain and underestimate dependence. ${ }^{52}$ Intraoperatively throughout the procedure, the necessary amount of opioid is made up of the preoperative daily dose of opioid taken regularly and the dose of opioid required by surgical stimulation. The most suitable opioids to administer to replace the opioid dose taken chronically appear to be long-acting opioids, since reasonably steady plasma concentrations of opioids are attainable. Frequently, the utilization of a continuous opioid infusion is the optimal method to deliver a stable serum concentration in cases where the oral route is not available perioperatively. Though the utilization of short-acting opioids can be sufficient for relieving short-lasting stimulation inflicted by the surgical procedure, their sole administration in chronic-pain patients on opioids may potentially cause withdrawal or inadequately controlled pain postoperatively. Postoperatively, it can be challenging to catch up on opioid dosing, due to unavoidable delays in procuring and giving opioids at the bedside, the potential hesitance of staff in the recovery room to dispense an adequately high dose of opioid, and the high intensity of pain that patients may feel. It must be remembered that patients who chronically take even modest amounts of opioids preoperatively (such as $<50 \mathrm{mg}$ /day oral morphine equivalents) will usually need their baseline opioid dose in addition to two- or more-fold the dose of opioids regularly given for well-controlled pain in opioid-naïve patients. ${ }^{35,50}$ It would be prudent to consider inpatient admission for patients with chronic use of opioids after ambulatory surgery, if presenting a challenge for pain control, for approximately 24 hours to determine the amount of oral opioid to be administered.

In chronically opioid-consuming patients, the shift to an oral opioid requires particular attentiveness. Inpatients need opioids for a comparatively extended duration of time epidurally or intravenously relative to those required in opioid-naïve patients. ${ }^{4}$ There is no widely acknowledged guideline as to the method in which postoperative transition to an oral opioid regimen is optimally accomplished in outpatients. Carroll et $\mathrm{al}^{3}$ recommended (for inpatients) converting the intravenous dose into its equivalent oral dose, giving half to two-thirds of this amount as a longacting opioid, and keeping the remaining dose available as an administered short-acting opioid as needed. In effect, short-acting opioids can address any breakthrough pain, and long-acting opioids can function as a stable baseline of pain management. As postoperative pain begins to diminish, reducing the dosage of a short-acting opioid seems to be an effective method in reducing the total dose of opioids administered daily.

Because such opioids as methadone with long half-lives can gradually accumulate, titration of the drugs should be done with care, in order to avoid overdose. Postoperatively, administering 1.5-fold the preoperative opioid dose orally, in addition to patient-controlled analgesic intravenous opioids for breakthrough pain, until the surgical pain begins to diminish has proved an effective method. Otherwise, patients can also be given the option of taking patientcontrolled intravenous opioids for the initial 24-48 hours postoperatively, during which opioid demands are altering most quickly. Following that window, the total amount of opioids given intravenously can be changed to a daily dose administered orally appropriate for managing the pain, as mentioned earlier. ${ }^{3}$

The perioperative management of patients who are chronically consuming opioids for pain management needs to be considered with caution for a number of reasons. First, administration of opioids continues to be an integral part of postoperative pain-management regimens, even with a history of significant chronic use. Also, the change from preoperative to postoperative opioids can be quite challenging to manage. It is also important to keep in mind that a sufficient dose of opioids needs to be sustained in order to avoid opioid withdrawal. ${ }^{3}$ Long-term administration of opioids may necessitate an increase in the dosage of opioids 
needed to maintain the appropriate level of analgesia. This phenomenon is most commonly attributed to the development of tolerance, but increases in dosage may also be explained by a variety of factors, including the advancement of the underlying disease that is causing the chronic pain. The literature has also demonstrated that patients on opioids chronically report greater postoperative pain, despite also consuming threefold more opioids postoperatively in a case-controlled retrospective study of 360 patients who had nonmalignant or malignant pain. ${ }^{5}$

\section{Interventions in patients with opioid-induced hypersensitivity}

An increasing number of studies suggest that long-term use of opioids causes changes in pain sensitivity. Recent literature offers a reason for the adverse effect of opioids, which must be taken into consideration: opioid-induced hyperalgesia. This body of literature asserts that the administration of opioids may prompt hypersensitivity to pain. ${ }^{3}$ Animal studies have shown that allodynia (pain caused by a stimulus that does not normally elicit pain) and hyperalgesia (heightened pain in response to a normally painful stimulus) can develop following continuous ${ }^{6}$ and repetitive ${ }^{7}$ administration of opioids. The literature also indicates that hyperalgesia is seen with acute opioid withdrawal and resolves rapidly following termination of the opioid administration. ${ }^{8}$ Studies with human subjects suggest that patients who are maintained on methadone are hyperalgesic in comparison to individuals who were previously addicted, ${ }^{9,10}$ and significantly more hyperalgesic than control subjects. ${ }^{12,13}$ Hyperalgesia has also been recorded in patients on other opioid-dependent populations and patients who are on slow-release oral morphine. ${ }^{14}$

Despite the extensive literature on opioid-induced hyperalgesia in patients maintained on methadone, there is minimal knowledge on the modifications of pain sensitivity in chronicpain patients given opioids, though studies suggest that these patients experience reformed sensitivity to acute pain, ${ }^{15,16}$ with hyperalgesia and allodynia being manifestations of neuropathic pain in the clinical setting. ${ }^{17}$ Notably, studies suggest that there is no significant difference in values of threshold of pain when patients treated with nonopioid analgesics are compared to patients with chronic pain receiving opioid treatment. ${ }^{18,19}$

However, an initial prospective pilot study based on patients with chronic low-back pain found that administration of oral morphine for a month was associated with decreased cold-pressor tolerance times. ${ }^{20}$ Hay et $\mathrm{al}^{21}$ conducted an observational study to determine whether pain sensitivity in patients maintained on methadone for dependence therapy was comparable to that in patients with noncancerous chronic pain on either morphine or methadone, relative to pain sensitivity reported in a control group via cold-pressor tests and electrical stimulation. The study found that chronic-pain patients managed with methadone and opioids demonstrated hyperalgesia when gauged by the cold-pressor test, but not by the electrical stimulation test.

\section{Interventions of wound injection and lavage}

Direct delivery of local analgesics to the surgical wound site can diminish postoperative opioid demands, and can be considered a management option when other regional methods are not appropriate. Subjects of a study by Goldstein et al, ${ }^{22}$ who underwent laparoscopic gynecological procedures and were given local anesthetics to their intraperitoneal wound site followed by wound lavage, utilized four to 17 times less opioid throughout the initial 24 hours postoperatively relative to patients who were given a saline placebo. ${ }^{22}$ Another study conducted on patients undergoing microdiskectomy and given direct local anesthetics at the surgical site did not observe any substantial opioid-sparing effects. ${ }^{23}$ Therefore, the success found in the literature in administering local anesthetics directly to the surgical site seemingly is dependent on the specific technique utilized for administration - the dose, concentration, and kind of local anesthetic given - and the kind of surgery being performed. ${ }^{3}$

\section{Interventions of regional anesthesia for opioid sparing}

The use of regional anesthesia provides numerous benefits, especially in patients taking opioids chronically. However, patients who have developed a dependence on opioids will still require regularly administered doses of opioid in order to avoid withdrawals and cover their chronic-pain needs, which may not be affected by the surgery. For instance, patients who chronically take opioids for low-back pain and are undergoing knee surgery will need analgesia for both after the surgical procedure. Studies have shown that the joint use of epidural opioids and intravenously administered opioids for breakthrough pain was adequate for counteracting any potential withdrawals. ${ }^{24-26}$ In a number of other studies on inpatients, the joint use of intravenously administered opioids and epidural opioids for breakthrough pain was adequate for counteracting any potential withdrawals. $3,4,27$ 
Opioid-sparing effects have been observed when a number of regional techniques have been employed, such as in the lower extremities, including femoral, lumbar plexus, paravertebral, and sciatic nerve blocks. Methods that utilize continuous catheters are especially attractive for delivering continuous pain control postoperatively. In the ambulatory setting, substantial opioid-sparing effects and optimal pain management have been demonstrated in patients given a continuous femoral nerve block that utilizes bupivacaine in those undergoing repair of the anterior cruciate ligament. ${ }^{28}$ It has been most commonly studied in the 3-in-1 and femoral nerve blocks. For instance, a study based on 1,338 patients who underwent total hip arthroplasty demonstrated analogous pain control in those treated with intravenous patient-controlled opioid analgesia, patient-controlled epidural analgesia, and those given a continuous 3-in-1 block. Of note, the patients who were administered a 3-in-1 block reported the best satisfaction. ${ }^{29}$ Substantial opioid-sparing effects and optimal pain management have been demonstrated in patients given a continuous femoral nerve block that utilizes bupivacaine in those undergoing repair of the anterior cruciate ligament. ${ }^{28}$ Comparable observations regarding the femoral block have been found in patients undergoing popliteal bypass surgery. ${ }^{30}$

For upper-extremity surgeries, opioid-sparing effects have been seen with the use of supraclavicular, interscalene, and axillary blocks. Though the majority of anesthesiologists are accustomed to single-bolus methods for peripheral nerve blocks, the insertion of catheters for extended administration of local anesthetics is becoming more common. Pain control has been found to be better in patients receiving blocks such as continuous interscalene or axillary blocks than in patients receiving intramuscular or intravenous opioids. There was improved patient satisfaction and reduced opioid consumption in the patients receiving blocks in the post operative period as compared to those patients receiving intramuscular or intravenous opioids. ${ }^{31,32}$ Though regional methods that utilize catheters can offer several days of pain management, injection of a single bolus may still be effective if insertion of a catheter is not possible. Other studies have observed opioid-sparing effects in single-injection methods in the immediate postoperative period. ${ }^{33,34}$

The use of regional anesthesia provides numerous benefits, and could be especially helpful in chronically opioid-consuming patients. For instance, patients who chronically take opioids for low-back pain and are undergoing knee surgery will need analgesia for both after the surgical procedure. A number of studies have reported cases of opioid withdrawal postoperatively when intrathecal opioids or epidurals were utilized solely for the purpose of perioperative pain management in patients who have developed an opioid dependence..$^{24,25} \mathrm{On}$ the other hand, in a number of other studies on inpatients, the joint use of intravenously administered opioids and epidural opioids for breakthrough pain was adequate for counteracting any potential withdrawals. ${ }^{27,35}$ Carroll et $\mathrm{al}^{3}$ asserted that routine day-to-day systemic delivery of minimally half of the dose of opioid given preoperatively is adequate to avoid withdrawal when regional anesthesia is utilized.

\section{Interventions to control postoperative nausea and vomiting}

In addition to pain, postoperative nausea and vomiting (PONV) are the most common complaints after general anesthesia. ${ }^{36}$ PONV could be due to a number of factors. These include pharmacological agents, such as inhalational anesthetics and opioids, patient-related factors, such as migraine, anxiety, motion sickness, and female sex, and the extent and nature of the surgery. Commonly used antiemetics include dopaminergic and cholinergic antagonists, such as prochlorperazine, promethazine, diphenhydramine, $5-\mathrm{HT}_{3}$ and $5-\mathrm{HT}_{4}$ serotonin blockers, and glucocorticoids. It is important preoperatively to identify patients at high risk of PONV, provide PONV prophylaxis with the most effective antiemetic single-therapy and combination-therapy regimens, including nonpharmacologic approaches and plan for strategies for treatment of $\mathrm{PONV}^{37}$ to prevent delay in discharge from hospital and unexpected admissions.

\section{Conclusion}

Notwithstanding the established acknowledgment that the management of postoperative pain is both undertreated and common, a significant number of patients continue to report moderate-to-severe pain postoperatively. ${ }^{38,39}$ Severe postoperative pain is associated with a greater risk of cardiovascular and pulmonary complications and longer recovery periods following inpatient procedures, and is the most commonly seen cause of unanticipated admissions to the hospital after ambulatory surgery and postponed discharge..$^{40,41}$

With the continuing rise in the number of ambulatory surgeries, attention to the perioperative pain management of patients in the ambulatory setting is crucial.

The intricacies of evaluation and optimum treatment of pain within a short period in the setting of ambulatory surgery of a variety of medical and sometimes serious conditions make optimum pain control in the ambulatory care setting truly challenging. The concept of an ambulatory pain-management provider overseeing care for patients with a history 
of pain issues, substance abuse, and/or chronic pain, as well as overseeing pain control in the PACU of the ambulatory surgery center, could be critical to the ever-growing community we serve. Ambulatory pain control can be highly complex, and is probably best accomplished through risk stratification, multidisciplinary communication, the use of multimodal analgesia, which can include regional anesthesia also, and other interventions when strategies fall short preoperatively.

There is a need for increased clinical research on quality of care in the ambulatory surgery setting, such as early intervention when problematic pain issues are recognized, in order to prevent emergency room visits, delayed discharges, and unplanned admissions. Interventions include control of anxiety, managing patients with a history of chronic pain, chronic opioid use, and the prevention of pain flare-ups in patients who are opioid-dependent and/or taking part in substance-abuse programs. New interventions will also be necessary when current multimodal analgesia modalities are insufficient or ineffective. It is always important to consider the cost of the interventions that may be needed after implementation of multimodal analgesia to make the strategies viable in the long term. Immediate hospital costs include services by personnel, supplies, and other resources. However, the cost of unplanned hospital admissions, returns to the emergency room for pain that is out of control, and the development of chronic pain as a result of poorly controlled acute pain is much more costly to the patient, society, and the health care system.

With the increasing need for optimum pain control in ambulatory surgery centers, the authors recommend a new concept wherein an ambulatory pain specialist oversees a clinical program of ambulatory pain control in each ambulatory center to facilitate the implementation of customized treatment plans to provide optimum pain control to all patients undergoing outpatient surgery. In addition, more research in the patterns and predictive factors of severe pain in ambulatory surgery and the multimodal management of acute surgically inflicted pain in patients with underlying chronic pain treated with opioids is needed.

\section{Disclosure}

The authors report no conflicts of interest in this work.

\section{References}

1. Dickerson DM. Acute pain management. Anesthesiol Clin. 2014;32(2): 495-504.

2. Dickerson DM. Pain management in the ambulatory setting. ASA Newsl. 2015;79(8):20-22.

3. Carroll IR, Angst MS, Clark JD. Management of perioperative pain in patients chronically consuming opioids. Reg Anesth Pain Med. 2004;29(6):576-591.
4. de Leon-Casasola OA. New concepts in postoperative pain management: is there more beyond pain control? J Fla Med Assoc. 1996;83(10):695-700.

5. Rapp SE, Ready LB, Nessly ML. Acute pain management in patients with prior opioid consumption: a case-controlled retrospective review. Pain. 1995;61(2):195-201.

6. Vanderah TW, Suenaga NM, Ossipov MH, Malan TP, Lai J, Porreca F. Tonic descending facilitation from the rostral ventromedial medulla mediates opioid-induced abnormal pain and antinociceptive tolerance. J Neurosci. 2001;21(1):279-286.

7. Mao J. Opioid-induced abnormal pain sensitivity: implications in clinical opioid therapy. Pain. 2002;100(3):213-217.

8. Li X, Angst MS, Clark JD. Opioid-induced hyperalgesia and incisional pain. Anesth Analg. 2001;93(1):204-209.

9. Compton MA. Cold-pressor pain tolerance in opiate and cocaine abusers: correlates of drug type and use status. J Pain Symptom Manage. 1994;9(7):462-473.

10. Ho A, Dole VP. Pain perception in drug-free and in methadone-maintained human ex-addicts. Proc Soc Exp Biol Med. 1979;162(3):392-395.

11. Gerbershagen HJ, Dagtekin O, Gaertner J, et al. Preoperative chronic pain in radical prostatectomy patients: preliminary evidence for enhanced susceptibility to surgically induced pain. Eur J Anaesthesiol. 2010;27(5):448-454.

12. Compton P, Charuvastra VC, Ling W. Pain intolerance in opioidmaintained former opiate addicts: effect of long-acting maintenance agent. Drug Alcohol Depend. 2001;63(2):139-146.

13. Doverty M, White JM, Somogyi AA, Bochner F, Ali R, Ling W. Hyperalgesic responses in methadone maintenance patients. Pain. 2001;90(1-2):91-96.

14. Mitchell TB, White JM, Somogyi AA, Bochner F. Switching between methadone and morphine for maintenance treatment of opioid dependence: impact on pain sensitivity and mood status. Am J Addict. 2006;15(4):311-315.

15. Cohen MJ, Naliboff BD, Schandler SL, Heinrich RL. Signal detection and threshold measures to loud tones and radiant heat in chronic low back pain patients and cohort controls. Pain. 1983;16(3):245-252.

16. Schmidt AJ, Brands AM. Persistence behavior of chronic low back pain patients in an acute pain situation. J Psychosom Res. 1986;30(3):339-346.

17. Jensen TS, Gottrup H, Sindrup SH, Bach FW. The clinical picture of neuropathic pain. Eur J Pharmacol. 2001;429(1-3):1-11.

18. Fillingim RB, Doleys DM, Edwards RR, Lowery D. Clinical characteristics of chronic back pain as a function of gender and oral opioid use. Spine (Phila Pa 1976). 2003;28(2):143-150.

19. Reznikov I, Pud D, Eisenberg E. Oral opioid administration and hyperalgesia in patients with cancer or chronic nonmalignant pain. Br J Clin Pharmacol. 2005;60(3):311-318.

20. Chu LF, Clark DJ, Angst MS. Opioid tolerance and hyperalgesia in chronic pain patients after one month of oral morphine therapy: a preliminary prospective study. J Pain. 2006;7(1):43-48.

21. Hay JL, White JM, Bochner F, Somogyi AA, Semple TJ, Rounsefell B. Hyperalgesia in opioid-managed chronic pain and opioid-dependent patients. J Pain. 2009;10(3):316-322.

22. Goldstein A, Grimault P, Henique A, Keller M, Fortin A, Darai E. Preventing postoperative pain by local anesthetic instillation after laparoscopic gynecologic surgery: a placebo-controlled comparison of bupivacaine and ropivacaine. Anesth Analg. 2000;91(2):403-407.

23. Mack PF, Hass D, Lavyne MH, Snow RB, Lien CA. Postoperative narcotic requirement after microscopic lumbar discectomy is not affected by intraoperative ketorolac or bupivacaine. Spine (Phila Pa 1976). 2001;26(6):658-661.

24. Tung AS, Tenicela R, Winter PM. Opiate withdrawal syndrome following intrathecal administration of morphine. Anesthesiology. 1980;53(4):340.

25. Messahel FM, Tomlin PJ. Narcotic withdrawal syndrome after intrathecal administration of morphine. Br Med J (Clin Res Ed). 1981;283(6289): $471-472$. 
26. Cousins MJ, Mather LE. Intrathecal and epidural administration of opioids. Anesthesiology. 1984;61(3):276-310.

27. de Leon-Casasola OA, Parker B, Lema MJ, Harrison P, Massey J. Postoperative epidural bupivacaine-morphine therapy: experience with 4,227 surgical cancer patients. Anesthesiology. 1994;81(2):368-375.

28. Tetzlaff JE, Andrish J, O'Hara J, Dilger J, Yoon HJ. Effectiveness of bupivacaine administered via femoral nerve catheter for pain control after anterior cruciate ligament repair. J Clin Anesth. 1997;9(7):542-545.

29. Singelyn FJ, Gouverneur JM. Postoperative analgesia after total hip arthroplasty: i.v. PCA with morphine, patient-controlled epidural analgesia, or continuous " 3 -in-1" block? A prospective evaluation by our acute pain service in more than 1,300 patients. J Clin Anesth. 1999;11(7):550-554.

30. Griffith JP, Whiteley S, Gough MJ. Prospective randomized study of a new method of providing postoperative pain relief following femoropopliteal bypass. Br J Surg. 1996;83(12):1735-1738.

31. Lehtipalo S, Koskinen LO, Johansson G, Kolmodin J, Biber B. Continuous interscalene brachial plexus block for postoperative analgesia following shoulder surgery. Acta Anaesthesiol Scand. 1999;43(3): 258-264.

32. Iskandar H, Rakotondriamihary S, Dixmérias F, Binje B, Maurette P. [Analgesia using continuous axillary block after surgery of severe hand injuries: self-administration versus continuous injection]. Ann Fr Anesth Reanim. 1998;17(9):1099-1103. French.

33. Borgeat A, Schäppi B, Biasca N, Gerber C. Patient-controlled analgesia after major shoulder surgery: patient-controlled interscalene analgesia versus patient-controlled analgesia. Anesthesiology. 1997;87(6): 1343-1347.

34. Muittari P, Kirvelä O. The safety and efficacy of intrabursal oxycodone and bupivacaine in analgesia after shoulder surgery. Reg Anesth Pain Med. 1998;23(5):474-478.

35. de Leon-Casasola OA, Lema MJ. Epidural sufentanil for acute pain control in a patient with extreme opioid dependency. Anesthesiology. 1992;76(5):853-856.

36. Becker DE. Nausea, vomiting, and hiccups: a review of mechanisms and treatment. Anesth Prog. 2010;57(4):150-156; quiz 157.

37. Gan TJ, Habib AS, Miller TE, White W, Apfelbaum JL. Incidence, patient satisfaction, and perceptions of post-surgical pain: results from a US national survey. Curr Med Res Opin. 2014;30(1):149-160.

38. Michel MZ, Sanders MK. Effectiveness of acute postoperative pain management. Br J Anaesth. 2003;91(3):448-449; author reply 9.
39. Dolin SJ, Cashman JN, Bland JM. Effectiveness of acute postoperative pain management: I. Evidence from published data. Br J Anaesth. 2002;89(3):409-423.

40. Tasmuth T, Kataja M, Blomqvist C, von Smitten K, Kalso E. Treatmentrelated factors predisposing to chronic pain in patients with breast cancer - a multivariate approach. Acta Oncol. 1997;36(6):625-630.

41. Shea RA, Brooks JA, Dayhoff NE, Keck J. Pain intensity and postoperative pulmonary complications among the elderly after abdominal surgery. Heart Lung. 2002;31(6):440-449.

42. Chapman CR, Davis J, Donaldson GW, Naylor J, Winchester D. Postoperative pain trajectories in chronic pain patients undergoing surgery: the effects of chronic opioid pharmacotherapy on acute pain. $J$ Pain. 2011;12(12):1240-1246.

43. Lee HJ, Yeomans DC. Opioid induced hyperalgesia in anesthetic settings. Korean J Anesthesiol. 2014;67(5):299-304.

44. Tompkins DA, Campbell CM. Opioid-induced hyperalgesia: clinically relevant or extraneous research phenomenon? Curr Pain Headache Rep. 2011;15(2):129-136.

45. Chapman CR, Donaldson GW, Davis JJ, Bradshaw DH. Improving individual measurement of postoperative pain: the pain trajectory. J Pain. 2011;12(2):257-262.

46. Celerier E, Laulin JP, Corcuff JB, Le Moal M, Simonnet G. Progressive enhancement of delayed hyperalgesia induced by repeated heroin administration: a sensitization process. $J$ Neurosci. 2001;21: 4074-4080.

47. Laulin JP, Celerier E, Larcher A, Le Moal M, Simonnet G. Opiate tolerance to daily heroin administration: an apparent phenomenon associated with enhanced pain sensitivity. Neuroscience. 1999;89(3):631-636.

48. Erlenwein J, Przemeck M2, Degenhart A, et al. The Influence of Chronic Pain on Postoperative Pain and Function After Hip Surgery: A Prospective Observational Cohort Study. J Pain. 2016;17(2):236-247.

49. Okifuji A, Hare BD. The association between chronic pain and obesity. J Pain Res. 2015;8: 399-408.

50. Tennant $F$. The physiologic effects of pain on the endocrine system. Pain Ther. 2013;2(2):75-86.

51. Kipping K, Maier C, Bussemas HH, Schwarzer A. Medication compliance in patients with chronic pain. Pain Physician. 2014;17(1) 81-94.

52. Savage R. Cyclo-oxygenase-2 inhibitors: when should they be used in the elderly? Drugs Aging. 2005;22(3):185-200.

\section{Journal of Pain Research}

\section{Publish your work in this journal}

The Journal of Pain Research is an international, peer reviewed, open access, online journal that welcomes laboratory and clinical findings in the fields of pain research and the prevention and management of pain. Original research, reviews, symposium reports, hypothesis formation and commentaries are all considered for publication.

\section{Dovepress}

The manuscript management system is completely online and includes a very quick and fair peer-review system, which is all easy to use. Visit http://www.dovepress.com/testimonials.php to read real quotes from published authors. 